\title{
Mechanism of texture development in ion-beam-assisted deposition
}

\author{
L. Dong and D. J. Srolovitz ${ }^{a}$ \\ Department of Materials Science and Engineering, The University of Michigan, Ann Arbor, \\ Michigan 48109-2136
}

(Received 25 January 1999; accepted for publication 2 June 1999)

\begin{abstract}
We perform a series of atomistic simulations of ion-beam-assisted deposition (IBAD) to identify the mechanism by which ion beams select crystallographic texture. Simulations were devised to distinguish between two previously proposed mechanisms: (1) preferential sputtering of differently oriented grains and (2) preferential damage of differently oriented grains followed by grain-boundary migration. We show that while preferential sputtering is capable of producing texture change, it does so much more slowly than observed in IBAD. Simulations that include only differential damage produce structures which are nearly indistinguishable from those seen in IBAD. These results conclusively demonstrate that texture evolution during ion-beam-assisted deposition is dominated by differential damage and subsequent recrystallization. (C) 1999 American Institute of Physics. [S0003-6951(99)02730-8]
\end{abstract}

Ion beams have been widely applied to modify film texture during the physical vapor deposition (PVD) of thin films. ${ }^{1-8}$ Two mechanisms have been proposed to explain how ion beams affect the crystallographic orientation of growing films. Bradley, Harper, and Smith ${ }^{9,10}$ developed a model for texture development based upon the variation of sputtering yield with crystal orientation. In their model, the variation of sputtering yield with grain orientation is associated with the existence of channeling directions, i.e., crystallographic directions along which ions easily penetrate into the crystal. Grains with channeling directions aligned parallel with the ion beam have a lower sputtering yield and, consequently, a higher growth rate than grains which are nonaligned with respect to the ion beam. This, combined with shadowing effects, leads to the oriented grains overgrowing the nonoriented ones and, hence, texture selection. This model is supported by experiments on a wide range of materials that demonstrate that the sputtering yield is indeed a strong function of the relative orientation of the grain and the ion beam (e.g., see Ref. 11).

Van Wyk and Smith ${ }^{1}$ studied the development of preferred orientation in evaporated $\mathrm{Cu}$ films which were subsequently bombarded with $40 \mathrm{keV} \mathrm{Cu}^{+}$ions. Upon ion bombardment, the texture of these films changed from strongly $\langle 111\rangle$ to $\langle 220\rangle$. They suggested that the $\langle 110\rangle$-oriented grains were damaged less by the ions than those with other orientations because $\langle 110\rangle$ is the easiest channeling direction in $\mathrm{Cu}$. They further argued that during the thermal spike associated with ion bombardment, the relatively perfect $\langle 110\rangle$ grains grow into their more damaged surroundings by a recrystallization process, thereby reorienting the more damaged material to $\langle 110\rangle$. Dobrev ${ }^{2}$ drew the same conclusions based on his observations of texture changes during $10 \mathrm{keV}$ $\mathrm{Ar}^{+}$bombardment of vapor-deposited fcc and hep metal films.

We previously performed a series of molecular dynamics simulations in which we saw clear evidence that an ion

a)Electronic mail: srol@umich.edu beam could effectively modify film texture during film growth. ${ }^{12}$ We were able to demonstrate that grains with their channeling direction aligned with the ion beam had a lower sputtering yield and suffered less damage than grains which were not aligned. This damage difference led to grainboundary migration in which the less damaged grains grew into the more damaged grains, in a process of recrystallization-induced film texturing. While this study clearly showed that both the sputtering yield differences and damage differences can lead to the evolution of film texture, as suggested in earlier work, it was not possible to conclude which effect dominates texture selection during IBAD. The goal of this letter is to determine which of the proposed mechanisms controls texture selection during IBAD.

A three-dimensional, molecular dynamics simulation program capable of performing both PVD and IBAD, described in an earlier publication, ${ }^{12}$ was employed in the present study. The interactions between atoms were described using the classical Lennard-Jones pair potential (characterized by a bond energy $\epsilon$ and bond length $r_{0}$ ) and the interactions between atoms and ions and between ions were described using the purely repulsive Molière potential (characterized only by a screening length and atomic number). ${ }^{13}$ The potential parameters were chosen to represent $\mathrm{Al}$ atoms and $\mathrm{Ne}$ ions. ${ }^{12}$ All length, energy, and time scales are reported in units associated with the parameters associated with the Al atom potentials: $r_{0}=0.286 \mathrm{~nm}$ (position of the minimum in the potential), $\epsilon=0.565 \mathrm{eV}$ (maximum depth of the atomic potential), $M=4.48 \times 10^{-26} \mathrm{~kg}$ (atomic mass), and $\tau=\sqrt{M r_{0}^{2} / \epsilon}=2.0 \times 10^{-13} \mathrm{~s}$.

We begin by simulating the ion-beam-assisted deposition of a bicrystal film in order to demonstrate that an ion beam does indeed modify the film orientation during deposition. The bicrystal was $24.8 d_{0}$ along the $x$ direction $\left(d_{0}\right.$ is the equilibrium lattice parameter at the simulation temperature), $15.59 d_{0}$ in the $y$ direction, and $6.5 d_{0}$ in the $z$ direction, and the grain boundaries lie along the $y-z$ plane at $x$ $=8.267$ and $x=16.534 d_{0}$ (note, periodic boundary conditions were employed in both the $x$ and $y$ directions). The 
temperature was thermostated at $0.4 \epsilon / k_{B}$, where the melting point is $\sim 0.7 \epsilon / k_{B}$ and $k_{B}$ is Boltzmann's constant. This temperature is high compared to typical growth conditions in order to accelerate diffusion processes so as to compensate for our relatively high deposition rate. One crystal was oriented with the [111] surface normal in the $z$ axis and the [110] and [ $\overline{1} \overline{1} 2]$ directions along the $x$ and $y$ axes, respectively. The other crystal was oriented with the [110], [110], and [001] directions along the $z, x$, and $y$ axes, respectively. The positions of the atoms in the bottom two layers of the simulation cell were fixed throughout the simulation in order to prevent the system from rotating or translating. The $\mathrm{Al}$ films were deposited with an energy of $2 \epsilon /$ atom and $E_{I}$ $=800 \epsilon /$ ion, an ion-to-atom arrival ratio of $R=1 / 2$, and an average atom deposition rate of 0.85 atom $/ \tau$. The ion relaxation time in the lattice is approximately $2 \tau,{ }^{12}$ which is slightly smaller than the mean ion arrival period employed. The ion beam was oriented perpendicular to the nominal surface (i.e., the $-z$ direction). [110] is the strongest channeling direction in this face-centered-cubic crystal ([111] is not a channeling direction in fcc crystals). The deposition flux was also oriented along the $-z$ direction. Figure 1 shows the atomic structure of the bicrystal following the deposition of $5300 \mathrm{Al}$ atoms. Note that although the simulations are three-dimensional, Fig. 1 is a projection along the $y$ direction, and hence, appears two-dimensional. We can clearly see that (1) the [111]-oriented grain has been overgrown by the [110] grain and (2) the film is considerably thinner above the [111] grain than above the [110] grain. While Fig. 1 definitively shows that [110] grains grow at the expense of [111] grains (although the [111] grain has a lower surface energy), and [110] out-of-plane texture is achieved, the two observations appear to support the operation of different mechanisms. The fact that the initial grain boundaries have migrated support the notion of recrystallization-induced texturing, while the existence of a thinner film above the [111] grain supports the notion that preferential sputtering dominates.

In order to distinguish between the effects of ioninduced differential sputtering and ion damage-induced recrystallization, several modifications to the simulation procedure were developed. In the first, we examined the role of differential sputtering without ion-induced damage. To this end, we performed a series of simulations on [111]- and [110]-oriented single crystals in order to extract the film growth rates under the same IBAD conditions (atom and ion-beam fluxes, energies, and orientations) employed in the simulations that led to Fig. 1. Under these conditions, the sputtering rate from the [111] surface was $\sim 1.4$ atoms/ion and $\sim 0.9$ atoms/ion from the [110] surface. For an ion-toatom arrival ratio of $R=1 / 2$, we found that $\sim 70 \%$ of the $\mathrm{Al}$ atoms that arrive on the [111] surface were sputtered away while $\sim 45 \%$ of the $\mathrm{Al}$ atoms that arrive on the [110] surface were sputtered away. Next, we modified the bicrystal IBAD simulation used to produce Fig. 1 by turning off the ion beam and randomly removing deposited $\mathrm{Al}$ atoms when they hit the [111] and [110] grain surfaces according to the sputtering rate found in the single-crystal IBAD simulations. The orientation of the surface where an atom lands is determined from the orientation of the majority of its neighboring atoms

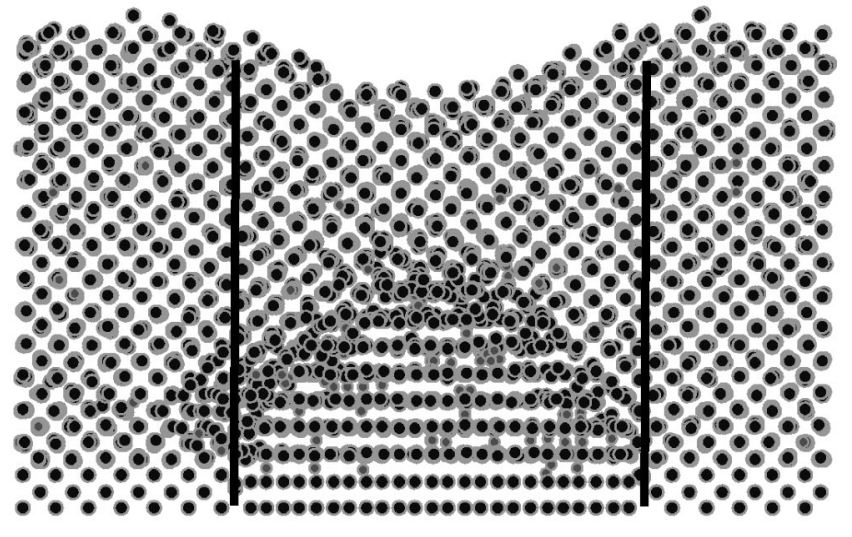

FIG. 1. Atomic structure of the bicrystal following ion-beam-assisted deposition. The ion-to-atom arrival ratio $R=1 / 2$ and the energy of the ion beam $E_{i}=800 \epsilon /$ ion. The two vertical lines indicate the locations of the two original grain boundaries. The smaller, light-gray particles are the ions that remain in the lattice (some escape into the gas).

(i.e., within $2.1 r_{0}$ ). In this way, the effects of ion-induced differential sputtering on growth rate were included, while the influence of the damage from the (relatively) high-energy ion beam was excluded.

The atomic structure of the bicrystal grown with the modified deposition simulation (including preferential sputtering effects and excluding ion damage) is shown in Fig. 2. Comparison of the actual grain-boundary positions with those in the starting structure (indicated by vertical lines in Fig. 2) demonstrates that the fraction of the bicrystal that is [111] oriented only slightly decreases during the deposition. This is likely associated with the attachment of the $(-z)$ deposited atoms to the sidewalls between the thicker grains and the vacuum above the thinner grains. This mechanism is the basis for differential sputtering creating texture change. ${ }^{14}$ The differences between this bicrystal structure and that from the IBAD simulation presented in Fig. 1 are striking. The IBAD bicrystal simulations in Fig. 1 show that the [111] grain is effectively pinched off and occluded from further growth with a similar number of deposited atoms. Therefore, we must conclude that while differential sputtering may lead to slow texture change, it cannot explain the relatively rapid texture change that is observed in IBAD.

Since differential sputtering alone is incapable of explaining the rapid texture changes observed during IBAD, we next examined whether differential damage alone can produce those texture changes. To this end, we modified the IBAD simulation procedure to exclude differential sputtering-induced growth rate differences while retaining the ion-beam-induced damage to the film. We did this by performing IBAD simulations identical with that which led to Fig. 1 (i.e., the same ion flux, ion energy, etc.), except we insured that both the [111] and [110] grains had exactly the same growth rate. Since the ion beam sputters $\mathrm{Al}$ atoms from the surface of the [111] grain at a higher rate than from the [110] grain surface, we randomly removed additional $\mathrm{Al}$ atoms from the [110] surface such that the effective growth rates (deposition minus sputtering minus removal rates) of [111] and [110] grains were identical. This additional removal rate was found from the sputtering simulations on single-crystal surfaces, as discussed above: the fraction of 


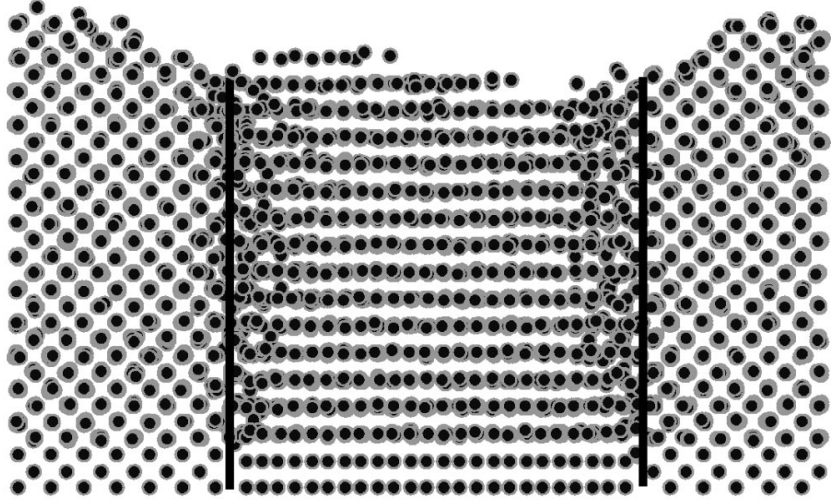

FIG. 2. Atomic structure of the bicrystal grown with modified deposition. Preferential sputtering effects are included and ion damage is excluded.

the deposited atoms removed are 0 and $1-[(1-0.7) /(1$ $-0.45)] \approx 0.455$ for the [111] and [110] grains, respectively.

The atomic structure of the bicrystal produced by the deposition simulations, which were modified to exclude differential sputtering differences while retaining ion-beaminduced damage, is shown in Fig. 3. The thickness of the entire bicrystal film is nearly uniform; consistent with the modification to the IBAD simulations. Figure 3 clearly shows that the [111] grain was pinched off/occluded by the adjacent [110] grain. Examination of the time evolution of the bicrystal structure (not shown) demonstrates that the two grain boundaries (on either side of the [111] grain) tilt towards the [111] grain during growth, meet while the film is still relatively thin, and then migrate towards the substrate. The tilting of the grain boundaries (they are pinned at the bottom of the substrate) is a defect-energy-driven grainboundary migration process akin to recrystallization. ${ }^{12}$ The postimpingement migration is driven both by the difference in stored defect (damage) energy between the two grains and by the grain-boundary curvature.

Comparison of Figs. 2 and 3 shows that while differential sputtering-induced height differences do lead to slow texture change (via occlusion of the grains that are preferentially sputtered), differential damage leading to grainboundary migration in a recrystallization-like process is a much more efficient mechanism for texture evolution. The degree to which differential damage-induced recrystallization dominates texture change during IBAD may be seen by comparison of the full IBAD simulations (Fig. 1) with the deposition-modified simulations that only include damage effects (Fig. 3). The only discernable difference between these two structures is that the film produced from the IBAD simulations is noticeably thinner above the occluded [111] grain. This is simply a result of preferential sputtering, discussed above. In both cases, the [111] grain was occluded by the surrounding [110] grain(s) at almost exactly the same film thickness.

In summary, we have performed a variety of atomistic simulations of ion-beam-assisted deposition in order to identify the dominant mechanism by which the ion beam modifies the crystallographic texture of growing films. The two

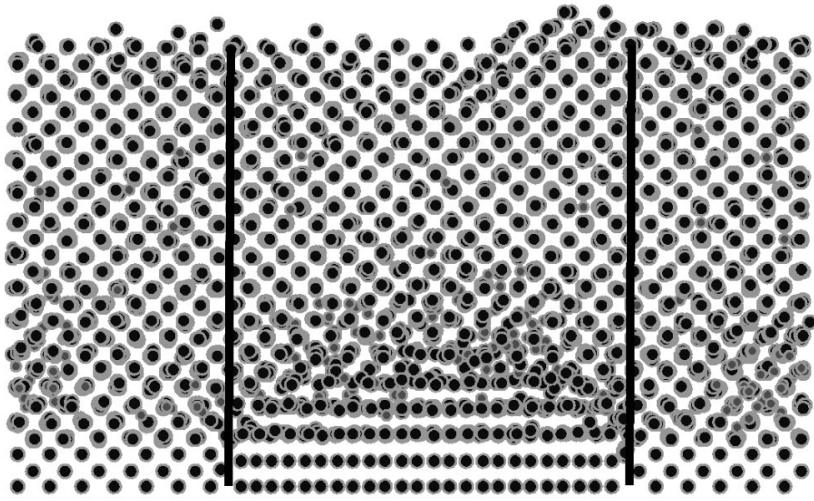

FIG. 3. Bicrystal grown with modified ion-beam-assisted deposition simulation under the same conditions as in Fig. 1. Ion damage is included and preferential sputtering effects are excluded.

competing mechanisms are: (1) preferential sputtering of differently oriented grains combined with shadowing and (2) differential damage of differently oriented grains followed by grain-boundary migration in a recrystallization-like process. Simulations were devised in which preferential sputtering occurs but with no damage and in which damage occurs while compensating for the preferential sputtering. These simulations clearly demonstrated that while preferential sputtering is capable of producing texture change, this texture change occurs much too slowly to explain that seen in IBAD simulations. On the other hand, the simulations that included only differential damage lead to structures which are nearly indistinguishable from those seen in IBAD simulations. Therefore, we conclude that texture evolution during ionbeam-assisted deposition is dominated by differential damage and subsequent recrystallization. Since recrystallization requires boundary migration, this mechanism cannot be operative in cases where the grain boundaries are immobile or exhibit very low mobility (e.g., in many ceramics). In this situation, the slower, preferential sputtering mechanism may dominate the texture evolution. Finally, we note that although these two mechanisms for texture selection during IBAD are quite distinct, both rely on the crystallographic nature of the channeling process.

${ }^{1}$ G. N. Van Wyk and H. J. Smith, Nucl. Instrum. Methods 170, 443 (1980). ${ }^{2}$ D. Dobrev, Thin Solid Films 92, 41 (1982).

${ }^{3}$ L. S. Yu, J. M. E. Harper, J. J. Cuomo, and D. A. Smith, Appl. Phys. Lett. 47, 932 (1985).

${ }^{4}$ L. S. Yu, J. M. E. Harper, J. J. Cuomo, and D. A. Smith, J. Vac. Sci. Technol. A 4, 443 (1986).

${ }^{5}$ W. Ensinger, Nucl. Instrum. Methods Phys. Res. B 106, 142 (1995).

${ }^{6}$ Y. Nagai, A. Tago, and T. Toshima, J. Vac. Sci. Technol. A 5, 61 (1987). ${ }^{7}$ G. Was, D. J. Srolovitz, Z. Ma, and L. Dong, Mater. Res. Soc. Symp. Proc. 441, 311 (1997)

${ }^{8}$ F. A. Smidt, Int. Mater. Rev. 35, 61 (1990).

${ }^{9}$ R. M. Bradley, J. M. E. Harper, and D. A. Smith, J. Appl. Phys. 60, 4160 (1986).

${ }^{10}$ R. M. Bradley, J. M. E. Harper, and D. A. Smith, J. Vac. Sci. Technol. A 5, 1792 (1987).

${ }^{11}$ H. E. Roosendaal, in Topics in Applied Physics, edited by R. Behrisch (Springer, Berlin, 1981), Vol. 47, Chap. 5.

${ }^{12}$ L. Dong and D. J. Srolovitz, J. Appl. Phys. 84, 5261 (1998).

${ }^{13}$ G. Molière, Z. Naturforsch. A 2A, 133 (1947).

${ }^{14}$ F. Ying, R. W. Smith, and D. J. Srolovitz, Appl. Phys. Lett. 61, 3007 (1996). 